Bull. Austral. Math. Soc.

VOL. $61(2000)$ [415-438]

\title{
NATURALITY AND INDUCED REPRESENTATIONS
}

\author{
Siegfried Echterhoff, S. KaliszewSKi, John QUigg and IAIN RAEBURN
}

We show that induction of covariant representations for $C^{*}$-dynamical systems is natural in the sense that it gives a natural transformation between certain crossedproduct functors. This involves setting up suitable categories of $C^{*}$-algebras and dynamical systems, and extending the usual constructions of crossed products to define the appropriate functors. From this point of view, Green's Imprimitivity Theorem identifies the functors for which induction is a natural equivalence. Various special cases of these results have previously been obtained on an ad hoc basis.

\section{Introduction}

Induced representations and the imprimitivity theorems which characterise them are a fundamental tool in the representation theory of dynamical systems and crossed products. In the powerful formulation of Rieffel and Green, induction is done by tensoring with a Hilbert bimodule, and the imprimitivity theorem tells us how to expand the left action to make this bimodule an imprimitivity bimodule. In recent applications, it has been necessary to know that this induction process is compatible with other constructions involving crossed products. Verifying such compatibility can be painful: it is often obvious that everything must work because "induction is natural", yet technically hard to sort out the details. And afterwards one is left feeling that one must have missed the point: the techniques are vaguely familiar even if the particular application isn't.

We have found that it is more satisfactory to phrase our questions and results directly in terms of Hilbert bimodules, rather than in terms of induced representations themselves. Thus in [10] we viewed a Hilbert $A-B$ bimodule $X$ as an arrow from $A$ to $B$, and said that a diagram

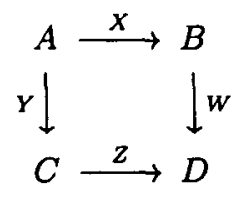

Received March 7, 2000

This research was partially supported by the Australian Research Council, and the Office of the Vice Provost for Research at Arizona State University.

Copyright Clearance Centre, Inc. Serial-fee code: 0004-9727/00 \$A2.00+0.00. 
of Hilbert bimodules "commutes in the strong sense" if $Y \otimes_{C} Z \cong X \otimes_{B} W$ as $A$ $D$ bimodules; if so, the Rieffel induction processes $\pi \mapsto X$ - Ind( $W$-Ind $\pi)$ and $\pi \mapsto$ $Y$ - $\operatorname{Ind}(Z-\operatorname{Ind} \pi)$ yield representations which are systematically equivalent in a way compatible with intertwining maps, direct-sum decompositions and continuity.

Here we make more precise the idea that we have been verifying the naturality of induction. Category theory tells us exactly what mathematicians should mean when they talk about naturality, and how we need to set things up to make sense of it. Here we build a category in which the objects are $C^{*}$-algebras, in which the morphisms are given by Hilbert bimodules, and in which the commutativity of a diagram like (1.1) says precisely that $Y \otimes_{C} Z \cong X \otimes_{B} W$. We then apply this framework to the induction process arising from Green's imprimitivity theorem [7, Proposition 3], proving that that theorem actually gives a natural transformation between certain crossed-product functors which take values in this category. This theorem includes as special cases a number of results in the recent literature which could be paraphrased as saying "Green induction is compatible with Morita equivalence".

Part of our point here is that taking the trouble to formulate theorems in this categorical framework will result in a theory which is more robust and more directly applicable. However, many applications require us to consider coactions or twisted crossed products, and setting up the appropriate categories, functors, and naturality theorems for these situations can take a good deal of technical effort. So in order to illustrate our approach, we concentrate here on one naturality theorem which requires only standard techniques, and leave equivariant versions involving coactions and twists to a more comprehensive sequel. We have tried hard to make this paper accessible to anyone familiar with the basic material on Hilbert modules and Morita equivalence implicit in the formulation of Green's theorem; this can be found, for example, in the early chapters of [16].

We begin in Section 2 by discussing our category $\mathcal{C}$. The objects in $\mathcal{C}$ will be $C^{*}$ algebras, a morphism from one $C^{*}$-algebra $A$ to another $B$ will be given by a Hilbert $A-B$ bimodule $X$, and the composition of morphisms will be given by the balanced tensor product of bimodules. There is asymmetry here: $X$ will be a right Hilbert $B$ module, and $A$ will act by adjointable operators on $X$, in the sense that there is a nondegenerate homomorphism $\kappa: A \rightarrow \mathcal{L}\left(X_{B}\right)$ describing the action. We call such a bimodule $X$ a right-Hilbert bimodule to stress that the Hilbert module structure is on the right. [These and related objects have been called Hilbert bimodules (for example, in [14]), or $C^{*}$-correspondences (for example, in [13]), but we have preferred to stick with the name used in [10] to stress that the Hilbert-module structure is on the right.] The morphisms will actually be isomorphism classes $\left[{ }_{A} X_{B}\right]$ of these right-Hilbert bimodules; this is necessary, because, for example, we expect ${ }_{B} B_{B}$ to give the identity morphism on $B$, so ${ }_{A} X_{B}$ and ${ }_{A}\left(X \otimes_{B} B\right)_{B}$ should define the same morphism in $\mathcal{C}$. In Section 2 we also show that the isomorphisms in $\mathcal{C}$ are exactly the (isomorphism classes of) imprimitivity 
bimodules, and prove that every morphism $\left[{ }_{A} X_{B}\right]$ in $\mathcal{C}$ can be factored as $\left[C Y_{B}\right] \circ[\varphi]$, where ${ }_{C} Y_{B}$ is an imprimitivity bimodule and $\varphi: A \rightarrow M(C)$ is a nondegenerate homomorphism.

In Section 3 we show how to view the crossed-product construction as a functor. We first have to add actions to the objects and morphisms of $\mathcal{C}$ to build a category $\mathcal{A}(G)$ whose objects are dynamical systems associated with a fixed locally compact group $G$; forming the crossed product then gives a functor $(A, \alpha) \mapsto A \times_{\alpha} G$ from $\mathcal{A}(G)$ to $\mathcal{C}$. Because the objects in $\mathcal{C}$ are $C^{*}$-algebras rather than isomorphism classes of $C^{*}$-algebras, we have to be careful to nominate a particular $C^{*}$-algebra as the crossed product. We discuss our nominee and its relationship to the universally defined crossed product which we usually prefer. Functoriality requires that we give a parallel construction of crossed products of Hilbert modules; the construction we have chosen is different from that of [2] and [1] and may be of some independent interest.

Our main theorem is proved in Section 4. Green's construction associates to each object $(A, \alpha)$ in $\mathcal{A}(G)$ and each closed subgroup $H$ of $G$ a right-Hilbert $\left(A \times_{\alpha} G\right)$ $\left(A \times_{\alpha} H\right)$ bimodule $X_{H}^{G}(A, \alpha)$; his Imprimitivity Theorem says that $X_{H}^{G}(A, \alpha)$ is also a $\left(C_{0}(G, A) \otimes_{\alpha \otimes \tau} G\right)-\left(A \times_{\alpha} H\right)$ imprimitivity bimodule. Our theorem says that the assignment $(A, \alpha) \mapsto\left[X_{H}^{G}(A, \alpha)\right]$ is a natural transformation. To prove this, we have to show that every morphism in $\mathcal{A}(G)$ gives rise to a certain commutative diagram in $\mathcal{C}$. We do this by factoring the morphism into an ordinary homomorphism and an imprimitivity bimodule; handling the homomorphism is straightforward, and we deal with the imprimitivity bimodule by adapting a powerful linking-algebra technique from [6, Section 4]. We find it intriguing that ideas developed to meet the demands of nonabelian duality are now feeding back into the theory of ordinary crossed products - both in the technical sense, as in our use of the linking-algebra technique, and in the motivational sense, in that we were led to formulate our theorem through our attempts to handle more complicated problems involving coactions.

\section{The Category $\mathcal{C}$}

Let $B$ be a $C^{*}$-algebra. A Hilbert $B$-module is a vector space $X$ which is a right $B$-module equipped with a positive definite $B$-valued sesquilinear form $\langle\cdot, \cdot\rangle_{B}$ satisfying

$$
\langle x, y \cdot b\rangle_{B}=\langle x, y\rangle_{B} b \text { and }\langle x, y\rangle_{B}^{*}=\langle y, x\rangle_{B}
$$

for all $x, y \in X$ and $b \in B$, and which is complete in the norm $\|x\|=\left\|\langle x, x\rangle_{B}\right\|^{1 / 2}$. For the general theory of Hilbert modules, we refer to [11] or [16].

Definition 2.1: Let $A$ and $B$ be $C^{*}$-algebras, and let $X_{B}$ be a Hilbert module which is full in the sense that $\overline{\langle X, X\rangle_{B}}=B$. We say that $X$ is a right-Hilbert $A-B$ bimodule if it is a nondegenerate left $A$-module satisfying

$$
a \cdot(x \cdot b)=(a \cdot x) \cdot b \text { and }\langle a \cdot x, y\rangle_{B}=\left\langle x, a^{*} \cdot y\right\rangle_{B}
$$


for all $a \in A, x, y \in X$, and $b \in B$. (This is equivalent to having a nondegenerate homomorphism $\kappa: A \rightarrow \mathcal{L}\left(X_{B}\right)$ and putting $a \cdot x=\kappa(a) x$.) An isomorphism of rightHilbert bimodules is a bijective linear map $\Phi:{ }_{A} X_{B} \rightarrow{ }_{A} Y_{B}$ such that

(i) $\Phi(a \cdot x)=a \cdot \Phi(x)$,

(ii) $\Phi(x \cdot b)=\Phi(x) \cdot b$, and

(iii) $\langle\Phi(x) \Phi(y)\rangle_{B}=\langle x, y\rangle_{B}$.

(Property (ii) is redundant - it follows from (iii) by considering $\|\Phi(x \cdot b)-\Phi(x) \cdot b\|^{2}-$ but we include it to emphasise that $\Phi$ is a bimodule homomorphism.)

EXAMPLE 2.2. If $B$ is a $C^{*}$-algebra, then $B$ becomes a right-Hilbert $B-B$ bimodule by putting

$$
a \cdot b \cdot c=a b c \text { and }\langle a, b\rangle_{B}=a^{*} b
$$

for $a, b, c \in B$. More generally, if $\varphi: A \rightarrow M(B)$ is a nondegenerate homomorphism, then $B_{B}$ becomes a right-Hilbert $A-B$ bimodule with left action given by

$$
a \cdot b=\varphi(a) b
$$

The morphisms from $A$ to $B$ will be the isomorphism classes of right-Hilbert $A$ $B$ bimodules (denoted with square brackets); we need to pass to isomorphism classes to show that composition of morphisms has the necessary properties. Two ordinary homomorphisms may give the same morphism in $\mathcal{C}$, but only if they differ by an inner automorphism:

PROPOSITION 2.3. Let $\varphi$ and $\psi$ be nondegenerate homomorphisms of $A$ into $M(C)$. Then $[\varphi]=[\psi]$ in $\mathcal{C}$ if and only if there exists $u \in U M(C)$ such that $\psi=\operatorname{Ad} u \circ \varphi$.

PRoOF: If $\psi=\operatorname{Ad} u \circ \varphi$, then the map $c \mapsto u c$ is a Hilbert-module automorphism of $C_{C}$ which intertwines the left actions coming from $\varphi$ and $\psi$.

For the converse, suppose $[\varphi]=[\psi]$ in $\mathcal{C}$, so there exists a linear bijection $L: C \rightarrow C$ such that

$$
L(c d)=L(c) d, \quad L(c)^{*} L(d)=c^{*} d, \quad \text { and } \quad L(\varphi(a) c)=\psi(a) L(c)
$$

for each $c, d \in C$. Define $R: C \rightarrow C$ by $R(c)=L^{-1}\left(c^{*}\right)^{*}$. Then the first two of these properties imply that $(L, R)$ is an invertible double centraliser of $C$, so there exists an invertible element $u$ of $M(C)$ such that $L(c)=u c$ for all $c$. Since

$$
u^{-1} c=L^{-1}(c)=L^{-1}\left(c^{* *}\right)^{* *}=R\left(c^{*}\right)^{*}=\left(c^{*} u\right)^{*}=u^{*} c
$$

it follows that $u$ is unitary, and $L(\varphi(a) c)=\psi(a) L(c)$ implies that $\psi=\operatorname{Ad} u \circ \varphi$.

To define composition we use the internal tensor product of Hilbert modules. Let ${ }_{A} X_{B}$ and ${ }_{B} Y_{C}$ be right-Hilbert bimodules. Then the algebraic tensor product $X \odot Y$ becomes a not-necessarily-complete right-Hilbert $A-C$ bimodule with actions given by

$$
a \cdot(x \otimes y)=a \cdot x \otimes y \quad \text { and } \quad(x \otimes y) \cdot b=x \otimes y \cdot b
$$


and a not-necessarily-definite $C$-valued inner product given by

$$
\langle x \otimes y, z \otimes w\rangle_{C}=\left\langle y,\langle x, z\rangle_{B} \cdot w\right\rangle_{C}
$$

[11, Proposition 4.5]. Modding out by the elements of length 0 and completing gives a right-Hilbert $A-C$ bimodule $X \otimes_{B} Y$ in which $x \cdot b \otimes y=x \otimes b \cdot y$. We emphasise that it is not obvious that the action of $a \in A$ on $X \odot Y$ extends to $X \otimes_{B} Y$; this requires a complete positivity argument as in [11, p. 42]. Note that $X \otimes_{B} Y$ is full, since $X$ and $Y$ are:

$$
\overline{\left\langle X \otimes_{B} Y, X \otimes_{B} Y\right\rangle_{C}}=\overline{\left\langle Y,\langle X, X\rangle_{B} \cdot Y\right\rangle_{C}}=\overline{\langle Y, B \cdot Y\rangle_{C}}=\overline{\langle Y, Y\rangle_{C}}=C .
$$

We call $X \otimes_{B} Y$ the balanced or internal tensor product.

PROPOSITION 2.4. There is a category $\mathcal{C}$ in which the objects are $C^{*}$-algebras, and in which the morphisms from $A$ to $B$ are the isomorphism classes of (full) rightHilbert $A-B$ bimodules. The composition of $[X]: A \rightarrow B$ with $[Y]: B \rightarrow C$ is the isomorphism class of the internal tensor product bimodule ${ }_{A}\left(X \otimes_{B} Y\right)_{C}$; the identity morphism on $A$ is the isomorphism class of the right-Hilbert bimodule ${ }_{A} A_{A}$.

REMARK 2.5. In any category, $\operatorname{Hom}(A, B)$ has to be a set for each pair of objects $A$ and $B$. This is not obviously true in $\mathcal{C}$ unless we limit the size of the bimodules involved. We can do this by considering only $C^{*}$-algebras and Hilbert modules with dense subsets whose cardinalities do not exceed a fixed large cardinal. For example, we could consider only separable $C^{*}$-algebras and bimodules. In practice, these issues should never present an unassailable problem, and we shall ignore them.

Proof of Proposition 2.4: We first claim that the composition of morphisms is well-defined. Suppose we have right-Hilbert bimodule isomorphisms $\Phi:{ }_{A} X_{B} \rightarrow{ }_{A} Z_{B}$ and $\Psi:{ }_{B} Y_{C} \rightarrow{ }_{B} W_{C}$. Then $\Phi \otimes \Psi: X \odot Y \rightarrow Z \otimes_{B} W$ is easily seen to preserve the actions and inner product, so extends to an isometric bimodule map $\Phi \otimes_{B} \Psi$. The map $\Phi^{-1} \otimes_{B} \Psi^{-1}$ is an inverse for $\Phi \otimes_{B} \Psi$, which is therefore a right-Hilbert $A-C$ bimodule isomorphism of $X \otimes_{B} Y$ onto $Z \otimes_{B} W$.

Next we establish that composition of morphisms in $\mathcal{C}$ is associative; it suffices to show that $X \otimes_{B}\left(Y \otimes_{C} Z\right)$ and $\left(X \otimes_{B} Y\right) \otimes_{C} Z$ are isomorphic for any right-Hilbert bimodules ${ }_{A} X_{B},{ }_{B} Y_{C}$, and ${ }_{C} Z_{D}$. But straightforward calculations show that the usual linear isomorphism of $X \odot(Y \odot Z)$ onto $(X \odot Y) \odot Z$ respects the module actions and right inner products, so extends to the desired isomorphism.

Finally, note that the maps $a \otimes x \mapsto a \cdot x$ and $y \otimes a \mapsto y \cdot a$ extend to isomorphisms $A \otimes_{A} X \cong X$ and $Y \otimes_{A} A \cong Y$ for any right-Hilbert bimodules ${ }_{A} X_{B}$ and ${ }_{B} Y_{A}$. Hence $\left[{ }_{A} A_{A}\right]$ is an identity morphism from $A$ to $A$ in $\mathcal{C}$.

It is implicit in Connes' definition of Morita equivalence [3, p. 155] that a morphism $[X]$ is invertible in $\mathcal{C}$ precisely when $X$ is an imprimitivity bimodule. This seemed obvious to us at first but we found it surprisingly hard to prove. 
By saying that a right-Hilbert bimodule ${ }_{A} X_{B}$ is an imprimitivity bimodule we mean that $X$ is also a (full) left-Hilbert $A$-module in such a way that

$$
{ }_{A}\langle x, y\rangle \cdot z=x \cdot\langle y, z\rangle_{B} \quad \text { and }{ }_{A}\langle x \cdot b, y\rangle={ }_{A}\left\langle x, y \cdot b^{*}\right\rangle \text {. }
$$

Equivalently, ${ }_{A} X_{B}$ is an imprimitivity bimodule if and only if the canonical map $\kappa: A \rightarrow$ $\mathcal{L}\left(X_{B}\right)$ induced by the left action of $A$ on $X_{B}$ is an isomorphism of $A$ onto the algebra $\mathcal{K}\left(X_{B}\right)$ of compact operators on $X_{B}$ [16, Proposition 3.8].

Proposition 2.6. Suppose ${ }_{A} X_{B}$ is a right-Hilbert bimodule. Then $[X]$ is an isomorphism in the category $\mathcal{C}$ if and only if $X$ is an $A-B$ imprimitivity bimodule.

One direction of Proposition 2.6 is easy: if ${ }_{A} X_{B}$ is an imprimitivity bimodule, and ${ }_{B} \widetilde{X}_{A}$ is its reverse bimodule, then the maps $x \otimes \tilde{y} \mapsto{ }_{A}\langle x, y\rangle$ and $\tilde{x} \otimes y \mapsto\langle x, y\rangle_{B}$ are isomorphisms of $X \otimes_{B} \tilde{X}$ onto ${ }_{A} A_{A}$ and $\widetilde{X} \otimes_{A} X$ onto ${ }_{B} B_{B}$, respectively (see [16, Proposition 3.28]), and hence $[\tilde{X}]$ is an inverse for $[X]$ in $\mathcal{C}$.

For the other direction we want to use a representation-theoretic argument, so we briefly discuss how right-Hilbert bimodules can be represented by bounded operators. These ideas are fairly standard: the analogues for imprimitivity bimodules appear in [5, Section 2].

DEFINITION 2.7: A representation $\left(\pi_{A}, \pi_{X}, \pi_{B}\right)$ of a right-Hilbert bimodule ${ }_{A} X_{B}$ is a triple consisting of nondegenerate representations $\pi_{A}$ and $\pi_{B}$ of $A$ and $B$ on Hilbert spaces $\mathcal{H}_{A}$ and $\mathcal{H}_{B}$, respectively, together with a linear map $\pi_{X}: X \rightarrow \mathcal{B}\left(\mathcal{H}_{B}, \mathcal{H}_{A}\right)$ such that

$$
\begin{aligned}
\text { (i) } & \pi_{X}(a \cdot x)=\pi_{A}(a) \pi_{X}(x), \\
\text { (ii) } & \pi_{X}(x \cdot b)=\pi_{X}(x) \pi_{B}(b), \text { and } \\
\text { (iii) } & \pi_{B}\left(\langle x, y\rangle_{B}\right)=\pi_{X}(x)^{*} \pi_{X}(y)
\end{aligned}
$$

for all $a \in A, x, y \in X$, and $b \in B$. (Again, property (ii) follows from property (iii), but we include it so the definition explicitly says that $\pi$ preserves the Hilbert-module structure of $X$.)

EXAMPLE 2.8. Suppose ${ }_{A} X_{B}$ is a right-Hilbert bimodule and $\pi_{B}$ is any nondegenerate representation of $B$ on a Hilbert space $\mathcal{H}_{B}$. The induced representation $\pi_{A}=X$-Ind $\pi_{B}$ of $A$ acts in $\mathcal{H}_{A}=X \otimes_{B} \mathcal{H}_{B}$ according to $\pi_{A}(a)(x \otimes \xi)=(a \cdot x) \otimes \xi[16$, Proposition 2.66]. We claim that there are bounded operators $\pi_{X}(x) \in \mathcal{L}\left(\mathcal{H}_{B}, \mathcal{H}_{A}\right)$ such that $\pi_{X}(x) \xi=x \otimes \xi$, and that $\left(\pi_{A}, \pi_{X}, \pi_{B}\right)$ is then a nondegenerate representation of ${ }_{A} X_{B}$ on $\left(\mathcal{H}_{A}, \mathcal{H}_{B}\right)$. Indeed, it is easy to check that $\pi_{X}(x)$ is bounded:

$$
\left\|\pi_{X}(x) \xi\right\|^{2}=(x \otimes \xi \mid x \otimes \xi)=\left(\pi_{B}\left(\langle x, x\rangle_{B}\right) \xi \mid \xi\right) \leqslant\left\|\pi_{B}\left(\langle x, x\rangle_{B}\right)\right\|\|\xi\|^{2}
$$

the relation $\pi_{A}(a) \pi_{X}(x)=\pi_{X}(a \cdot x)$ is immediate from the definition of $\pi_{A}$; the relation $\pi_{X}(x) \pi_{B}(b)=\pi_{X}(x \cdot b)$ follows from the balancing property $(x \cdot b) \otimes \xi=x \otimes \pi_{B}(b) \xi$ of the tensor product; and the definition of the inner product on $X \otimes_{B} \mathcal{H}_{B}$ implies that $\pi_{B}\left(\langle x, y\rangle_{B}\right)=\pi_{X}(x)^{*} \pi_{X}(y)$. 
In this example, the representation $\pi_{A}$ is by definition the restriction of a representation $\pi_{\mathcal{L}}$ of the algebra $\mathcal{L}\left(X_{B}\right)$ of adjointable operators on $X$ (strictly speaking, $\left.\pi_{A}=\pi_{\mathcal{L}} \circ \kappa\right)$. The following observation will be useful in the proof of Proposition 2.6.

Leмma 2.9. Let $X_{B}$ be a right Hilbert module, let $\pi_{B}$ be a faithful representation of $B$ on $\mathcal{H}_{B}$, and consider the representation $\left(\pi_{\mathcal{L}}, \pi_{X}, \pi_{B}\right)$ of the right-Hilbert bimodule $\mathcal{L}(X) X_{B}$ discussed in Example 2.8. Then $\pi_{\mathcal{L}}$ is faithful, and

$$
\pi_{\mathcal{L}}\left(\mathcal{K}\left(X_{B}\right)\right)=\pi_{X}(X) \pi_{X}(X)^{*}:=\overline{\operatorname{span}}\left\{\pi_{X}(x) \pi_{X}(y)^{*} \mid x, y \in X\right\} .
$$

Proof: By definition, $\mathcal{K}\left(X_{B}\right)$ is the closed span of the rank-one operators $\left\{\Theta_{x, y}\right\}$ $x, y \in X\}$ [16, Definition 2.24], so it is enough to show that $\pi_{\mathcal{L}}\left(\Theta_{x, y}\right)=\pi_{X}(x) \pi_{X}(y)^{*}$. We can verify this by applying both sides to vectors of the form $x \otimes \xi$, which densely $\operatorname{span} X \otimes_{B} \mathcal{H}_{B}$. We then have

$$
\begin{aligned}
\pi_{\mathcal{L}}\left(\Theta_{x, y}\right)(z \otimes \xi) & =\Theta_{x, y}(z) \otimes \xi=x \cdot\langle y, z\rangle_{B} \otimes \xi=x \otimes \pi\left(\langle y, z\rangle_{B}\right) \xi \\
& =x \otimes \pi_{X}(y)^{*} \pi_{X}(z) \xi=\pi_{X}(x)\left(\pi_{X}(y)^{*} \pi_{X}(z) \xi\right) \\
& =\left(\pi_{X}(x) \pi_{X}(y)^{*}\right)(z \otimes \xi)
\end{aligned}
$$

as required.

Since $\pi_{B}$ is faithful and $X$ is a $\mathcal{K}\left(X_{B}\right)-B$ imprimitivity bimodule, the induced representation $\pi_{\mathcal{K}}$ is faithful (this follows from the Rieffel correspondence [16, Proposition 3.24]), and hence so is its extension $\pi_{\mathcal{L}}$ to $\mathcal{L}\left(X_{B}\right)=M\left(\mathcal{K}\left(X_{B}\right)\right)$.

Proof of Proposition 2.6: We have to show that if there is a right-Hilbert bimodule ${ }_{B} Y_{A}$ such that $X \otimes_{B} Y \cong{ }_{A} A_{A}$ and $Y \otimes_{A} X \cong{ }_{B} B_{B}$ as right-Hilbert bimodules, then $X$ is an $A-B$ imprimitivity bimodule (and in fact $Y$ will then be isomorphic to $\tilde{X}$ ). We choose a faithful nondegenerate representation $\pi_{B}$ of $B$ on $\mathcal{H}_{B}$, and use the inducing construction of Example 2.8 to give a representation $\left(\pi_{A}, \pi_{X}, \pi_{B}\right)$ of ${ }_{A} X_{B}$ on $\left(\mathcal{H}_{B}, \mathcal{H}_{A}\right)$ with $\mathcal{H}_{A}=X \otimes_{B} \mathcal{H}_{B}$. We shall prove the result by showing that the presence of $Y$ implies that $\pi_{A}$ is an isomorphism of $A$ onto the image $\overline{\operatorname{span}}\left\{\pi_{X}(x) \pi_{X}(y)^{*}\right\}$ of the algebra $\mathcal{K}\left(X_{B}\right)$ of compact operators.

We begin by noting that because $A$ acts faithfully on itself and ${ }_{A} A_{A} \cong X \otimes_{B} Y, A$ must act faithfully on $X$ : if $a \cdot x=0$ for all $x$, then $a \cdot(x \otimes y)=0$ for all $y$, whence $a b=0$ for all $b \in A$, and $a=0$. Thus $\kappa: A \rightarrow \mathcal{L}\left(X_{B}\right)$ is injective, and the injectivity of $\pi_{\mathcal{L}}$ implies that $\pi_{A}=\pi_{\mathcal{L}} \circ \kappa$ is faithful. To show that $\pi_{A}$ has the right image, we construct a representation $\left(\pi_{B}, \sigma, \pi_{A}\right)$ of $Y$ on $\left(\mathcal{H}_{A}, \mathcal{H}_{B}\right)$.

Let $\rho: Y \rightarrow \mathcal{B}\left(\mathcal{H}_{A}, Y \otimes_{A} \mathcal{H}_{A}\right)$ be the representation induced from $\pi_{A}$, given as usual by $\rho(y) \zeta=y \otimes \zeta$. By assumption, there exists a right-Hilbert bimodule isomorphism $\Psi: Y \otimes_{A} X \rightarrow B$, which we use to define a unitary $U: Y \otimes_{A} \mathcal{H}_{A}=Y \otimes_{A} X \otimes_{B} \mathcal{H}_{B} \rightarrow \mathcal{H}_{B}$ by

$$
U(y \otimes x \otimes \xi)=\pi_{B}(\Psi(y \otimes x)) \xi
$$


Now we define

$$
\sigma(y)=U \circ \rho(y)
$$

For $y \in Y, b \in B$, and $x \otimes \xi \in \mathcal{H}_{A}$ we get

$$
\begin{aligned}
\sigma(b \cdot y)(x \otimes \xi) & =U(b \cdot y \otimes x \otimes \xi)=\pi_{B}(\Psi(b \cdot y \otimes x)) \xi \\
& =\pi_{B}(b) \pi_{B}(\Psi(y \otimes x)) \xi=\pi_{B}(b) U(y \otimes x \otimes \xi) \\
& =\pi_{B}(b) \sigma(y)(x \otimes \xi),
\end{aligned}
$$

and we also have

$$
\sigma(y)^{*} \sigma(z)=\rho(y)^{*} U^{*} U \rho(z)=\rho(y)^{*} \rho(z)=\pi_{A}\left(\langle y, z\rangle_{A}\right)
$$

for all $y, z \in Y$, since $\rho$ has right coefficient map $\pi_{A}$. Thus $\left(\pi_{B}, \sigma, \pi_{A}\right)$ is indeed a rightHilbert bimodule representation of $Y$. In particular, we have $\pi_{A}(A)=\sigma(Y)^{*} \sigma(Y)$.

It only remains to show that $\sigma(Y)^{*}=\pi_{X}(X)$. For this we observe that for $y \in Y$, $x \in X$, and $\xi \in \mathcal{H}_{B}$ we have

$$
\sigma(y) \pi_{X}(x) \xi=\sigma(y)(x \otimes \xi)=\pi_{B}(\Psi(y \otimes x)) \xi
$$

hence $\sigma(Y) \pi_{X}(X)=\pi_{B}\left(\Psi\left(Y \otimes_{A} X\right)\right)=\pi_{B}(B)$. Using $\pi_{A}(A)=\sigma(Y)^{*} \sigma(Y)$, it now follows that

$$
\begin{aligned}
\pi_{X}(X)= & \pi_{X}(A \cdot X)=\pi_{A}(A) \pi_{X}(X)=\sigma(Y)^{*} \sigma(Y) \pi_{X}(X) \\
& =\sigma(Y)^{*} \pi_{B}(B)=\sigma(Y \cdot B)^{*}=\sigma(Y)^{*}
\end{aligned}
$$

This completes the proof of Proposition 2.6.

Proposition 2.10. For every morphism $\left[{ }_{A} X_{B}\right]$ in $\mathcal{C}$, there exists a $C^{*}$-algebra $C$, a nondegenerate homomorphism $\varphi: A \rightarrow M(C)$, and an imprimitivity bimodule ${ }_{C} Y_{B}$ such that

$$
\left[{ }_{A} X_{B}\right]=\left[{ }_{C} Y_{B}\right] \circ[\varphi] \text { in } \mathcal{C}
$$

In other words, such that ${ }_{A} X_{B} \cong{ }_{A}\left(C \otimes_{C} Y\right)_{B}$ as a right-Hilbert bimodule.

Proof: Let $C=\mathcal{K}\left(X_{B}\right)$ and let $Y=X$ viewed as a $C-B$ imprimitivity bimodule (see [16, Proposition 3.8]). Since $\mathcal{L}\left(X_{B}\right) \cong M\left(\mathcal{K}\left(X_{B}\right)\right)$ [16, Corollary 2.54], we can view the canonical nondegenerate homomorphism $\kappa: A \rightarrow \mathcal{L}\left(X_{B}\right)$ as a nondegenerate homomorphism $\varphi: A \rightarrow M\left(\mathcal{K}\left(X_{B}\right)\right)$. The map $c \otimes y \mapsto c \cdot y$ extends to an isomorphism of $C \otimes_{C} Y$ onto $X$. 


\section{Crossed-Product Functors}

Our next goal is to formalise the idea that assignments like $(A, \alpha) \mapsto A \times_{\alpha} G$ are functors into $\mathcal{C}$; this requires that we construct an equivariant category $\mathcal{A}(G)$ in which the objects are dynamical systems $(A, G, \alpha)$.

Definition 3.1: Let $G$ be a locally compact group, let ${ }_{A} X_{B}$ be a right-Hilbert bimodule, and let $\alpha$ and $\beta$ be (strongly continuous) actions of $G$ on $A$ and $B$. An $(\alpha, \beta)$ compatible action of $G$ on $X$ is a homomorphism $\gamma$ of $G$ into the group of invertible linear transformations on $X$ such that

(i) $\gamma_{s}(a \cdot x)=\alpha_{s}(a) \cdot \gamma_{s}(x)$

(ii) $\gamma_{s}(x \cdot b)=\gamma_{s}(x) \cdot \beta_{s}(b)$

(iii) $\left\langle\gamma_{s}(x), \gamma_{s}(y)\right\rangle_{B}=\beta_{s}\left(\langle x, y\rangle_{B}\right)$

for each $s \in G, a \in A, x, y \in X$, and $b \in B$; and such that each map $s \mapsto \gamma_{s}(x)$ is continuous from $G$ into $X$. (As usual, (ii) follows from (iii).) Two $(\alpha, \beta)$-compatible actions $\gamma$ and $\eta$ on $X$ and $Y$ are equivariantly isomorphic if there exists an isomorphism $\Phi$ of $X$ onto $Y$ such that $\Phi\left(\gamma_{s}(x)\right)=\eta_{s}(\Phi(x))$ for each $s \in G$ and $x \in X$.

EXAMPLE 3.2. If $(A, \alpha)$ and $(B, \beta)$ are actions of $G$, and $\varphi: A \rightarrow M(B)$ is equivariant in the sense that $\varphi\left(\alpha_{s}(a)\right)=\beta_{s}(\varphi(a))$, then $\beta$ is an $(\alpha, \beta)$-compatible action of $G$ on the bimodule ${ }_{A} B_{B}$ of Example 2.2.

PROPOSITION 3.3. Let $G$ be a locally compact group.

(i) There is a category $\mathcal{A}(G)$ in which the objects are $C^{*}$-algebras with actions of $G$, and in which the morphisms from $(A, \alpha)$ to $(B, \beta)$ are the equivariant isomorphism classes of (full) right-Hilbert $A-B$ bimodules with $(\alpha, \beta)$ compatible actions of $G$. The composition of $[X, \gamma]:(A, \alpha) \rightarrow(B, \beta)$ with $[Y, \eta]:(B, \beta) \rightarrow(C, \varepsilon)$ is the isomorphism class of the tensor product action $\left(X \otimes_{B} Y, \gamma \otimes_{B} \eta\right)$; the identity morphism on $(A, \alpha)$ is $\left[(A, \alpha)(A, \alpha)_{(A, \alpha)}\right]$.

(ii) $\left[\left({ }_{A, \alpha)}(X, \gamma)_{(B, \beta)}\right]\right.$ is an isomorphism in $\mathcal{A}(G)$ if and only if ${ }_{A} X_{B}$ is an imprimitivity bimodule and $\alpha_{s}\left({ }_{A}\langle x, y\rangle\right)={ }_{A}\left\langle\gamma_{s}(x), \gamma_{s}(y)\right\rangle$.

(iii) For every morphism $\left[{ }_{(A, \alpha)}(X, \gamma)_{(B, \beta)}\right]$ in $\mathcal{A}(G)$, there exists an isomorphism $\left[(C, \varepsilon)(Y, \eta)_{(B, \beta)}\right]$ and an $\alpha-\varepsilon$ equivariant nondegenerate homomorphism $\varphi: A \rightarrow M(C)$ such that

$$
[X, \gamma]=[Y, \eta] \circ[\varphi, \varepsilon] \text { in } \mathcal{A}(G) .
$$

Proof: Adding actions to Propositions 2.4, 2.6, and 2.10 is routine, except possibly in statement (iii). In the proof of Proposition 2.10, we took $C=\mathcal{K}\left(X_{B}\right)$ and $\varphi=\kappa: A \rightarrow$ $\mathcal{L}\left(X_{B}\right)=M(C)$, so we need to show that $\gamma$ induces an action on $\mathcal{K}\left(X_{B}\right)$. But for each $T \in \mathcal{L}\left(X_{B}\right), \varepsilon_{s}(T): x \mapsto \gamma_{s}\left(T\left(\gamma_{s}^{-1}(x)\right)\right)$ is an adjointable operator with $\varepsilon_{s}(T)^{*}=\varepsilon_{s}\left(T^{*}\right)$, and in fact $T \mapsto \varepsilon_{s}(T)$ is an automorphism of $\mathcal{L}\left(X_{B}\right)$. A quick calculation shows that

$$
\varepsilon_{s}\left({ }_{\alpha}\langle x, y\rangle\right)={ }_{\mathcal{K}}\left\langle\gamma_{s}(x), \gamma_{s}(y)\right\rangle,
$$


so $\varepsilon_{s}$ restricts to an automorphism of $\mathcal{K}\left(X_{B}\right)$. Since $G$ acts continuously on $X, \varepsilon$ gives an action of $G$ on $\mathcal{K}$, and (3.1) says that ${ }_{(G, \varepsilon)}(X, \gamma)_{(B, \beta)}$ is an isomorphism in $\mathcal{A}(G)$. Now recall that $\kappa\left(\alpha_{s}(a)\right)(x)=\alpha_{s}(a) \cdot x=\gamma_{s}\left(a \cdot \gamma_{s}^{-1}(x)\right)$ to see that $\varphi$ is $\alpha-\varepsilon$ equivariant; it is then easy to check that the isomorphism $c \otimes y \mapsto c \cdot y$ of $C \otimes_{C} Y$ onto $X$ is $\varepsilon \otimes \eta-\gamma$ equivariant.

To construct the crossed product of a system $(A, G, \alpha)$, we begin with the vector space $C_{c}(G, A)$ of continuous functions $f: G \rightarrow A$ of compact support. This is a *-algebra with the operations

$$
f * g(s)=\int_{G} f(t) \alpha_{t}\left(g\left(t^{-1} s\right)\right) d t \quad \text { and } \quad f^{*}(s)=\alpha_{s}\left(f\left(s^{-1}\right)^{*}\right) \Delta_{G}(s)^{-1} .
$$

A covariant representation of $(A, G, \alpha)$ on $\mathcal{H}$ is a pair $(\pi, U)$ consisting of a nondegenerate representation $\pi: A \rightarrow \mathcal{B}(\mathcal{H})$ and a unitary representation $U: G \rightarrow U(\mathcal{H})$ such that $\pi\left(\alpha_{s}(a)\right)=U_{s} \pi(a) U_{s}^{*}$ for $s \in G$ and $a \in A$. Routine calculations show that there is a *-representation $\pi \times U$ of $C_{c}(G, A)$ on $\mathcal{H}$, called the integrated form of $(\pi, U)$, such that

$$
\pi \times U(f) \xi=\int_{G} \pi(f(s)) U_{s} \xi d s
$$

for all $\xi \in \mathcal{H}$. (Inserting the vector $\xi$ is technically helpful because it makes the integrand norm-continuous.) Since $\|\pi \times U(f)\| \leqslant \int_{G}\|f(s)\| d s$ for every $f$, we can define a seminorm on $C_{c}(G, A)$ by

$$
\|f\|_{*}=\sup \{\|\pi \times U(f)\|:(\pi, U) \text { is a covariant representation of }(A, G, \alpha)\} ;
$$

it is a norm because the regular representation $\tilde{\pi} \times \lambda$ on $L^{2}\left(G, \mathcal{H}_{\pi}\right)$ is faithful on $C_{c}(G, A)$ if $\pi$ is faithful on $A$. [There is ostensibly a set-theoretic problem here since it is not obvious that we are taking the supremum over a well-defined set of real numbers. But the numbers of the form $\|\pi \times U(f)\|$ do form a subclass of $\mathbf{R}$, and every subclass of a set is again a set [12, Proposition 4.6].]

We now define the crossed product $A \times_{\alpha} G$ to be the completion of $C_{c}(G, A)$ in the norm $\|\cdot\|_{*}$. (For this to be a construction of a particular $C^{*}$-algebra rather than an isomorphism class of $C^{*}$-algebras, we must be clear that forming completions is a construction, but this can be achieved by defining the completion of a normed space $X$ to be the closure of $X$ in its double dual.) Notice that every representation $\pi \times U$ of $C_{c}(G, A)$ is by definition continuous for $\|\cdot\|_{*}$, and hence extends to a representation of $A \times{ }_{\alpha} G$, which we continue to denote by $\pi \times U$.

Remark 3.4. In [15], a crossed product for the system $(A, G, \alpha)$ is any $C^{*}$-algebra $B$ equipped with a nondegenerate homomorphism $i_{A}: A \rightarrow M(B)$ and a strictly continuous homomorphism $i_{G}: G \rightarrow U M(B)$ satisfying

(a) $\left(i_{A}, i_{G}\right)$ is covariant, in the sense that $i_{A}\left(\alpha_{s}(a)\right)=i_{G}(s) i_{A}(a) i_{G}(s)^{*}$ for all $a \in A$ and $s \in G$; 
(b) if $(\pi, U)$ is a covariant representation, there is a nondegenerate representation $\varphi=\varphi_{\pi, U}$ of $B$ such that $\pi=\varphi \circ i_{A}$ and $U=\varphi \circ i_{G}$; and

(c) the elements $i_{A} \times i_{G}(f)=\int_{G} i_{A}(f(s)) i_{G}(s) d s$ for $f \in C_{c}(G, A)$ span a dense subspace of $B$.

From our present perspective, we can view these axioms as properties which allow us to identify isomorphic copies of $A \times_{\alpha} G$. To be more precise, if $\left(B, i_{A}, i_{G}\right)$ is a crossed product in the sense of [15], then we claim that $i_{A} \times i_{G}$ extends to an isomorphism of $A \times{ }_{\alpha} G$ onto $B$.

To see this, first represent $B$ on Hilbert space, so that by $(\mathrm{a}),\left(i_{A}, i_{G}\right)$ becomes a covariant representation of $(A, G, \alpha)$. It follows from the definition of $\|\cdot\|_{*}$ that $i_{A} \times i_{G}$ extends to a representation of $A \times{ }_{\alpha} G$, and then (c) implies that $i_{A} \times i_{G}$ maps $A \times{ }_{\alpha} G$ onto (the represented copy of) $B$. Now choose any covariant representation $(\pi, U)$ of $(A, G, \alpha)$. Then by (b), $\pi \times U(f)=\varphi_{\pi, U}\left(i_{A} \times i_{G}(f)\right)$ for $f \in C_{c}(G, A)$, and hence for all $f \in A \times{ }_{\alpha} G$. But this implies $\|\pi \times U(f)\| \leqslant\left\|i_{A} \times i_{G}(f)\right\|$, and hence that $\|f\|_{*} \leqslant\left\|i_{A} \times i_{G}(f)\right\|$. Thus $i_{A} \times i_{G}$ is injective, which proves the claim.

We next seek to define crossed products of right-Hilbert bimodules. There are several possible approaches; for example, we could deduce much of the following proposition from the construction of [2, Section 5] for imprimitivity bimodules by first factoring the right-Hilbert bimodule as in Proposition 3.3(iii). We have opted for a direct treatment partly because it is more elementary, and partly because we feel that the details should be available. This is also the approach used by Kasparov to construct crossed-product Hilbert modules for the study of the $K K$-theory of group $C^{*}$-algebras in [9, Section 6 , Definition 1].

Proposition 3.5. Let $\gamma$ be an $(\alpha, \beta)$-compatible action of $G$ on a rightHilbert bimodule ${ }_{A} X_{B}$. There exists a right-Hilbert $\left(A \times_{\alpha} G\right)-\left(B \times_{\beta} G\right)$ bimodule $X \times_{\gamma} G$, which contains $C_{c}(G, X)$ as a dense subspace, and which satisfies

$$
\begin{aligned}
f \cdot h(s) & =\int_{G} f(t) \cdot \gamma_{t}\left(h\left(t^{-1} s\right)\right) d t, \\
h \cdot g(s) & =\int_{G} h(t) \cdot \beta_{t}\left(g\left(t^{-1} s\right)\right) d t, \quad \text { and } \\
\langle h, k\rangle_{B \times_{B} G}(s) & =\int_{G} \beta_{t^{-1}}\left(\langle h(t), k(t s)\rangle_{B}\right) d t
\end{aligned}
$$

for $f \in C_{c}(G, A), h, k \in C_{c}(G, X)$, and $g \in C_{c}(G, B)$.

LEMMA 3.6. Let $\pi \times U$ be a representation of $B \times{ }_{\beta} G$ on a Hilbert space $\mathcal{H}$. Then for each $h, k \in C_{c}(G, X)$ and $\xi, \eta \in \mathcal{H}$, (3.4) satisfies

$$
\left(\pi \times U\left(\langle k, h\rangle_{B \times G}\right) \xi \mid \eta\right)=\left(\omega_{h, \xi} \mid \omega_{k, \eta}\right)
$$

where $\omega_{h, \xi}=\int_{G} h(s) \otimes U_{s} \xi d s \in X \otimes_{B} \mathcal{H}$. 
ProOF: We have

$$
\begin{aligned}
\left(\pi \times U\left(\langle k, h\rangle_{B \times G}\right) \xi \mid \eta\right) & =\left(\int_{G} \pi\left(\langle k, h\rangle_{B \times G}(s)\right) U_{s} \xi d s \mid \eta\right) \\
& =\left(\int_{G} \pi\left(\int_{G} \beta_{t-1}\left(\langle k(t), h(t s)\rangle_{B}\right) d t\right) U_{s} \xi d s \mid \eta\right) \\
& =\int_{G} \int_{G}\left(U_{t}^{*} \pi\left(\langle k(t), h(t s)\rangle_{B}\right) U_{t s} \xi \mid \eta\right) d s d t \\
& =\int_{G} \int_{G}\left(\pi\left(\langle k(t), h(s)\rangle_{B}\right) U_{s} \xi \mid U_{t} \eta\right) d s d t \\
& =\int_{G} \int_{G}\left(h(s) \otimes U_{s} \xi \mid k(t) \otimes U_{t} \eta\right) d s d t \\
& =\left(\int_{G} h(s) \otimes U_{s} \xi d s \mid \int_{G} k(t) \otimes U_{t} \eta d t\right) \\
& =\left(\omega_{h, \xi} \mid \omega_{k, \eta}\right) .
\end{aligned}
$$

Proof of Proposition 3.5: We begin by showing that $C_{c}(G, X)$ can be completed to give a Hilbert $B \times{ }_{\beta} G$-module $X \times{ }_{\gamma} G$ satisfying (3.3) and (3.4). Straightforward calculations show that (3.3) and (3.4) make $C_{c}(G, X)$ into a pre-inner product $B \times{ }_{\beta} G$ module, so we need only verify that the sesquilinear form of (3.4) is positive definite.

To do so, fix, for the remainder of the proof, a faithful representation $\pi \times U$ of $B \times{ }_{\beta} G$ on a Hilbert space $\mathcal{H}$. Then for each $\xi \in \mathcal{H}$, Lemma 3.6 gives

$$
\left(\pi \times U\left(\langle h, h\rangle_{B \times G}\right) \xi \mid \xi\right)=(\omega \mid \omega) \geqslant 0,
$$

which shows positivity. For definiteness, suppose $h \in C_{c}(G, X)$ satisfies $\langle h, h\rangle_{B \times G}=0$. Then Lemma 3.6 gives $\left(\pi \times U\left(\langle h, h\rangle_{B \times G}\right) \xi \mid \xi\right)=(\omega \mid \omega)=0$ for each $\xi \in \mathcal{H}$, so that $\omega=\int_{G} h(s) \otimes U_{s} \xi d s=0$ for each $\xi$. Thus,

$$
\left(\int_{G} h(s) \otimes U_{s} \xi d s \mid x \otimes \zeta\right)=\left(\int_{G} \pi\left(\langle x, h(s)\rangle_{B}\right) U_{s} \xi d s \mid \zeta\right)=\left(\pi \times U\left(g_{x}\right) \xi \mid \zeta\right)=0
$$

for each $\xi, \zeta \in \mathcal{H}$ and $x \in X$, where $g_{x}(s)=\langle x, h(s)\rangle_{B}$ defines $g_{x} \in C_{c}(G, X)$. It follows that $g_{x}=0$ for all $x \in X$, whence $h=0$ in $C_{c}(G, X)$.

We next show that the $\left(B \times_{\beta} G\right)$-valued inner product on $X \times_{\gamma} G$ is full. Since $C_{c}(G, B)$ has an approximate identity for $B \times{ }_{\beta} G$, since $\langle X, X\rangle_{B}$ is dense in $B$, and since $B$ acts nondegenerately on $B \times_{\beta} G$, functions of the form $f^{*}\langle x, y\rangle_{B} g$, where $f, g \in C_{c}(G, B)$ and $x, y \in X$, span a dense subspace of $B \times{ }_{\beta} G$. Now letting $(x \cdot f)(s)=x \cdot(f(s))$ define $x \cdot f \in C_{c}(G, X)$, a straightforward calculation shows that $\langle x \cdot f, y \cdot g\rangle_{B \times G}=f^{*}\langle x, y\rangle_{B} g$, so that $\left\langle C_{c}(G, X), C_{c}(G, X)\right\rangle_{B \times G}$ is dense in $B \times_{\beta} G$.

We now claim that (3.2) makes $X \times_{\gamma} G$ into a right-Hilbert $\left(A \times_{\alpha} G\right)-\left(B \times_{\beta} G\right)$ bimodule. Again, checking the algebraic conditions of Definition 2.1 at the level of $C_{c^{-}}$ functions is routine; we need to show that $\|f \cdot h\| \leqslant\|f\|\|h\|$ for $f \in C_{c}(G, A)$ and $h \in C_{c}(G, X)$ to see that this extends to an action of $A \times_{\alpha} G$ on $X \times_{\gamma} G$. 
To show this, we begin by defining actions of $A$ and $G$ on $C_{c}(G, X)$ by

$$
(a \cdot h)(s)=a \cdot(h(s)) \text { and }(t \cdot h)(s)=\gamma_{t}\left(h\left(t^{-1} s\right)\right) .
$$

Now we use these actions to define

$$
\rho(a)(h \otimes \xi)=a \cdot h \otimes \xi \quad \text { and } \quad V_{t}(h \otimes \xi)=t \cdot h \otimes \xi ;
$$

we claim that $\rho(a)$ is bounded on $C_{\mathfrak{c}}(G, X) \odot \mathcal{H}$, and hence extends to an operator on $\left(X \times_{\gamma} G\right) \otimes_{B \times_{\beta} G} \mathcal{H}$. Again using Lemma 3.6, and writing $\omega_{i}$ for $\omega_{h_{i}, \xi_{i}}$, we have

$$
\begin{aligned}
\left\|\rho(a)\left(\sum_{i=1}^{n} h_{i} \otimes \xi_{i}\right)\right\|^{2} & =\left(a \cdot \sum_{i=1}^{n} h_{i} \otimes \xi_{i} \mid a \cdot \sum_{j=1}^{n} h_{j} \otimes \xi_{j}\right) \\
& =\sum_{i, j=1}^{n}\left(a \cdot h_{i} \otimes \xi_{i} \mid a \cdot h_{j} \otimes \xi_{j}\right) \\
& =\sum_{i, j=1}^{n}\left(\pi \times U\left(\left\langle a \cdot h_{j}, a \cdot h_{i}\right\rangle_{B \times G}\right) \xi_{i} \mid \xi_{j}\right) \\
& =\sum_{i, j=1}^{n}\left(\int_{G} a \cdot h_{i}(s) \otimes U_{s} \xi_{i} d s \mid \int_{G} a \cdot h_{j}(s) \otimes U_{s} \xi_{j} d s\right) \\
& =\sum_{i, j=1}^{n}\left(a \cdot \omega_{i} \mid a \cdot \omega_{j}\right) \\
& =\left(a \cdot \sum_{i=1}^{n} \omega_{i} \mid a \cdot \sum_{j=1}^{n} \omega_{j}\right) \\
& \leqslant\|a\|^{2} \sum_{i, j=1}^{n}\left(\omega_{i} \mid \omega_{j}\right) \\
& =\|a\|^{2} \sum_{i, j=1}^{n}\left(\pi \times U\left(\left\langle h_{j}, h_{i}\right\rangle_{B \times G}\right) \xi_{i} \mid \xi_{j}\right) \\
& =\|a\|^{2}\left(\sum_{i=1}^{n} h_{i} \otimes \xi_{i} \mid \sum_{j=1}^{n} h_{j} \otimes \xi_{j}\right) \\
& =\|a\|^{2}\left\|\sum_{i=1}^{n} h_{i} \otimes \xi_{i}\right\|^{2},
\end{aligned}
$$

where the inequality holds because $A$ acts boundedly on $X \otimes_{B} \mathcal{H}$ via the induced representation $X$-Ind $\pi$.

It is straightforward to check that each $V_{t}$ is unitary, and then that $(\rho, V)$ is covariant for $(A, G, \alpha) ; \rho$ is nondegenerate because $A$ acts nondegenerately on $X$, so we get a nondegenerate representation $\rho \times V$ of $A \times_{\alpha} G$ on $\left(X \times_{\gamma} G\right) \otimes_{B \times G} \mathcal{H}$. 
Now for $f \in C_{c}(G, A), h \in C_{c}(G, X)$, and $\xi \in \mathcal{H}$, we have

$$
\rho \times V(f)(h \otimes \xi)=\int_{G} \rho(f(t)) V_{t}(h \otimes \xi) d t=\left(\int_{G} f(t) t \cdot h d t\right) \otimes \xi=f \cdot h \otimes \xi,
$$

so

$$
\begin{aligned}
\left(\pi \times U\left(\langle f \cdot h, f \cdot h\rangle_{B \times G}\right) \xi \mid \xi\right) & =(f \cdot h \otimes \xi \mid f \cdot h \otimes \xi) \\
& =(\rho \times V(f)(h \otimes \xi) \mid \rho \times V(f)(h \otimes \xi)) \\
& \leqslant\|f\|^{2}(h \otimes \xi \mid h \otimes \xi) \\
& =\left(\pi \times U\left(\|f\|^{2}\langle h, h\rangle_{B \times G}\right) \xi \mid \xi\right) .
\end{aligned}
$$

It follows that $\|f \cdot h\| \leqslant\|f\|\|h\|$.

Finally, to see that the action of $A \times_{\alpha} G$ on $X \times_{\gamma} G$ is nondegenerate, note that for $h \in C_{c}(G, X)$, the Cauchy-Schwarz inequality gives

$$
\begin{aligned}
\|h\|^{2} & =\left\|\langle h, h\rangle_{B \times G}\right\| \\
& \leqslant \int_{G}\left\|\langle h, h\rangle_{B \times G}(s)\right\| d s \\
& \leqslant \int_{G} \int_{G}\left\|\beta_{t^{-1}}\langle h(t), h(t s)\rangle_{B}\right\| d t d s \\
& \leqslant \int_{G} \int_{G}\|h(t)\|\|h(t s)\| d t d s \\
& \leqslant\left(\int_{G}\|h(t)\| d t\right)^{2},
\end{aligned}
$$

so $\|h\| \leqslant\|h\|_{1}$. Now standard arguments show that we can choose $f \in C_{c}(G, A)$ to make $\|h-f \cdot h\|_{1}$ arbitrarily small: take $f$ of the form $s \mapsto a \chi(s)$ as $a$ runs through an approximate identity for $A$ and $\chi$ runs through an approximate identity for $C_{c}(G)$.

EXAMPLE 3.7. Suppose $(A, \alpha)$ and $(B, \beta)$ are objects in $\mathcal{A}(G)$, and $\varphi: A \rightarrow M(B)$ is a nondegenerate homomorphism such that $\varphi \circ \alpha_{s}=\beta_{s} \circ \varphi$. Then the crossed-product morphism ${ }_{A} B_{B} \times G$ is the completion of $C_{c}(G, B)$ in the norm coming from $B \times{ }_{\beta} G$, and hence is precisely $\left(B \times_{\beta} G\right)_{B \times} G$; the left action is given by a nondegenerate homomorphism $\varphi \times G: A \times_{\alpha} G \rightarrow M\left(B \times{ }_{\beta} G\right)$. Equation 3.2 shows that for functions $f \in C_{c}(G, A)$ and $g \in C_{c}(G, B)$, we have

$$
((\varphi \times G)(f) g)(s)=\int_{G} \varphi(f(t)) \beta_{t}\left(g\left(t^{-1} s\right)\right) d t .
$$

Proposition 3.8. The maps defined by

$$
(A, \alpha) \mapsto A \times_{\alpha} G \text { and }\left[(A, \alpha)(X, \gamma)_{(B, \beta)}\right] \mapsto\left[{ }_{A \times G}\left(X \times_{\gamma} G\right)_{B \times G}\right]
$$

give a functor from $\mathcal{A}(G)$ to $\mathcal{C}$. 
Proof: We first show that the map on morphisms is well-defined. Suppose $\varphi: X \rightarrow Y$ is an isomorphism of right-Hilbert $A-B$ bimodules which is equivariant for $(\alpha, \beta)$-compatible actions $\gamma$ and $\eta$ of $G$. Then $\Phi(h)(s)=\varphi(h(s))$ is easily seen to give a bijective map $\Phi: C_{c}(G, X) \rightarrow C_{c}(G, Y)$ which respects the right-Hilbert bimodule structures (Equations 3.2-3.4) and hence extends to a right-Hilbert $\left(A \times_{\alpha} G\right)-\left(B \times{ }_{\beta} G\right)$ bimodule isomorphism of $X \times_{\gamma} G$ onto $Y \times_{\eta} G$. Example 3.7 shows that identity morphisms go to identity morphisms.

It only remains to see that the assignment $[X, \gamma] \mapsto\left[X \times_{\gamma} G\right]$ respects composition of morphisms; that is, if $\left({ }_{A} X_{B}, \gamma\right)$ is $(\alpha, \beta)$-compatible and $\left({ }_{B} Y_{C}, \eta\right)$ is $(\beta, \varepsilon)$-compatible, we need to show that

$$
\left(X \times_{\gamma} G\right) \otimes_{B \times G}\left(Y \times_{\eta} G\right) \cong\left(X \otimes_{B} Y\right) \times_{\gamma \otimes_{\eta}} G
$$

as right-Hilbert $A \times_{\alpha} G-C \times{ }_{\varepsilon} G$ bimodules. [An analog of this result appears in [8, Lemma $3.10]$, in the context of not-necessarily-full Hilbert modules.]

The rule

$$
\Psi(h \otimes k)(s)=\int_{G} h(t) \otimes \eta_{t}\left(k\left(t^{-1} s\right)\right) d t
$$

defines a linear map from $C_{c}(G, X) \odot C_{c}(G, Y)$ to $C_{c}\left(G, X \otimes_{B} Y\right)$ which preserves the preright-Hilbert bimodule structures. In order to see that $\Psi$ extends to an isomorphism of the completions, we need only verify that $\Psi$ has dense range for the inductive limit topology. For this, let $x \in X$ and $f \in C_{c}(G, B)$, and define $h \in C_{c}(G, X)$ by $h(s)=x \cdot f(s)$. Then for $k \in C_{c}(C, Y)$ we have

$$
\begin{aligned}
\Phi(h \otimes k)(s) & =x \otimes \int_{G} f(t) \cdot \eta_{t}\left(k\left(t^{-1} s\right)\right) d t \\
& =x \otimes(f \cdot k)(s) .
\end{aligned}
$$

Now, we can approximate $k$ by $f \cdot k$ in the inductive limit topology, and taking $k$ of the form $k(s)=y g(s)$ for $y \in Y$ and $g \in C_{c}(G)$ we can thus approximate the function $s \mapsto(x \otimes y) g(s)$. But such functions have inductive-limit-dense span in $C_{c}\left(G, X \otimes_{B} Y\right)$. D

\section{NATURALITY IN GREEN'S IMPRIMITIVITY THEOREM}

Suppose that $\alpha$ is an action of a locally compact group $G$ on a $C^{*}$-algebra $A$, and $H$ is a closed subgroup of $G$. Takesaki showed in [17] how Mackey's construction of induced representations of groups could be modified to induce covariant representations of $(A, H, \alpha)$ to representations of $(A, G, \alpha)$. Subsequently Green showed how the integrated forms of Takesaki's induced representations could be obtained using Rieffel's abstract induction by Hilbert bimodules [7]. To construct his $\left(A \times_{\alpha} H\right)-\left(A \times_{\alpha} G\right)$ bimodule 
$X_{H}^{G}(A, \alpha)$, Green completed the $C_{c}(G, A)-C_{c}(H, A)$ bimodule $C_{c}(G, A)$ in which

$$
\begin{aligned}
f \cdot x(t) & =\int_{G} f(s) \alpha_{s}\left(x\left(s^{-1} t\right)\right) \Delta(s)^{1 / 2} d s \\
x \cdot g(t) & =\int_{H} x(t h) \alpha_{t h}\left(g\left(h^{-1}\right)\right) \Delta(h)^{-1 / 2} d h \\
\langle x, y\rangle_{A \times_{\alpha} H}(h) & =\int_{G} \alpha_{s}\left(x\left(s^{-1}\right)^{*} y\left(s^{-1} h\right)\right) \Delta(h)^{-1 / 2} d s
\end{aligned}
$$

for $x \in C_{c}(G, A), f \in C_{c}(G, A) \subset A \times_{\alpha} G, g \in C_{c}(H, A)$. The representation of $A \times_{\alpha} G$ induced from a representation $\pi$ of $A \times_{\alpha} H$ is then by definition the representation $X_{H}^{G}$-Ind $\pi$ acting in $X_{H}^{G} \otimes_{A \times H} \mathcal{H}_{\pi}$.

Imprimitivity theorems tell us how to recognise induced representations. In Rieffel's modern formulation, we recognise a representation which has been induced from $H$ by the presence of an auxilary representation of $C_{0}(G / H)$. In Green's realisation, this representation comes from the action of $C_{0}(G / H)$ on $C_{c}(G, A)$ given by

$$
c \cdot x(t)=c(t H) x(t)
$$

and the crucial properties of this action are that it commutes with the left action of $A$ on $X_{H}^{G}$ and is covariant for the left action of $G$ : more formally,

$$
c \cdot\left(i_{A}(a) \cdot x\right)=i_{A}(a) \cdot(c \cdot x) \text { and } i_{G}(s) \cdot\left(c \cdot\left(i_{G}(s)^{*} \cdot x\right)\right)=\tau_{s}(c) \cdot x,
$$

where we have extended the left action of $A \times_{\alpha} G$ on $X_{H}^{G}$ to $M\left(A \times_{\alpha} G\right)$ and $\tau$ is the action of $G$ on $C_{0}(G / H)$ given by $\tau_{s}(c)(t)=c\left(s^{-1} t\right)$. In other words, the left actions combine to give an action of $\left(A \otimes C_{0}(G / H)\right) \times_{\alpha \otimes \tau} G$ on $X_{H}^{G}(A, \alpha)$, and it is the content of Green's Imprimitivity Theorem that $X_{H}^{G}$ is an $\left(\left(A \otimes C_{0}(G / H)\right) \times_{\alpha \otimes \tau} G\right)-\left(A \times_{\alpha} H\right)$ imprimitivity bimodule. Note that the left action of $A \times_{\alpha} G$ on $X_{H}^{G}$ factors through the left action of $\left(A \otimes C_{0}(G / H)\right) \times_{\alpha \otimes \tau} G$ via the canonical map of $A \times G$ into $M\left(\left(A \otimes C_{0}(G / H)\right) \times_{\alpha \otimes \tau} G\right)$.

THEOREM 4.1. Suppose $H$ is a closed subgroup of a locally compact group $G$. Then the assignment $(A, \alpha) \mapsto\left[X_{H}^{G}(A, \alpha)\right]$ is a natural transformation between the functors $(A, \alpha) \mapsto A \times_{\alpha} G$ and $(A, \alpha) \mapsto A \times_{\alpha} H$ from $\mathcal{A}(G)$ to $\mathcal{C}$, and a natural equivalence between the functors $(A, \alpha) \mapsto\left(A \otimes C_{0}(G / H)\right) \times_{\alpha \otimes \tau} G$ and $(A, \alpha) \mapsto A \times_{\alpha} H$ from $\mathcal{A}(G)$ to $\mathcal{C}$.

Before we begin the proof, we point out that we have implicitly asserted in the statement of the theorem that the crossed products $A \times_{\alpha} H$ and $\left(A \otimes C_{0}(G / H)\right) \times{ }_{\alpha \otimes \tau} G$ define functors on $\mathcal{A}(G)$. The first case is quite easy: we just need to check that restricting every action in sight from $G$ to $H$ is a functor from $\mathcal{A}(G)$ to $\mathcal{A}(H)$, and then compose this with the crossed-product functor from $\mathcal{A}(H)$ to $\mathcal{C}$. For the second, we need to prove that $(A, \alpha) \mapsto\left(A \otimes C_{0}(G / H), \alpha \otimes \tau\right)$ is a functor from $\mathcal{A}(G)$ to itself, or, more generally, that for any given action $\varepsilon$ of $G$ on a $C^{*}$-algebra $C,(A, \alpha) \mapsto\left(A \otimes_{\min } C, \alpha \otimes \varepsilon\right)$ is a functor. 
This is non-trivial: a morphism $\left[{ }_{A} X_{B}, \gamma\right]$ goes to a morphism $[X \otimes C, \gamma \otimes \varepsilon]$ based on the external tensor product $A \otimes C(X \otimes C)_{B \otimes C}$ of bimodules (see [16, Corollary 3.38]). But all the details are routine, so we shall omit them.

A natural transformation $T$ between two functors $F, G: \mathcal{A} \rightarrow \mathcal{B}$ assigns to each object $A$ of $\mathcal{A}$ a morphism $T(A): F(A) \rightarrow G(A)$ such that, for every morphism $\varphi: A \rightarrow B$ in $\mathcal{A}$, the diagram

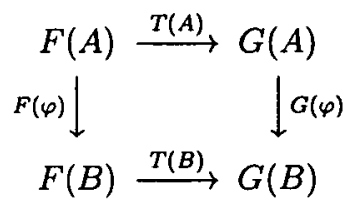

commutes in $\mathcal{B}$. The transformation $T$ is a natural equivalence if $T(A)$ is an isomorphism for all objects $A$. Green's Theorem tells us that $\left[X_{H}^{G}(A, \alpha)\right]$ is a morphism from $A \times_{\alpha} G$ to $A \times_{\alpha} H$ and an isomorphism from $\left(A \otimes C_{0}(G / H)\right) \times_{\alpha \otimes \tau} G$ to $A \times_{\alpha} H$, so we need to show that for every morphism $\left[_{(A, \alpha)}(X, \gamma)_{(B, \beta)}\right]$ in the category $\mathcal{A}(G)$, the diagrams

$$
\begin{array}{cc}
A \times_{\alpha} G \stackrel{X_{H}^{B}(A, \alpha)}{\longrightarrow} A \times_{\alpha} H \\
\left.X \times_{\gamma} G\right\rfloor & \downarrow \times_{\gamma} H \\
B \times_{\beta} G \stackrel{X_{H}^{G}(B, \beta)}{\longrightarrow} B \times_{\beta} H
\end{array}
$$

and

$$
\begin{array}{cc}
\left(A \otimes C_{0}(G / H)\right) \times \times_{\alpha \otimes \tau} G \stackrel{X_{H}^{G}(A, \alpha)}{\longrightarrow} A \times_{\alpha} H \\
\left(X \otimes C_{0}(G / H)\right) \times \gamma \otimes r \\
\left(B \otimes C_{0}(G / H)\right) \times_{\beta \otimes \tau} G \stackrel{X_{H}^{G}(B, \beta)}{\longrightarrow} B \times_{\beta} H
\end{array}
$$

commute in $\mathcal{C}$.

ProOF OF ThEOREM 4.1: We establish the commutativity of both (4.1) and (4.2) by factoring $[X, \gamma]=[Y, \eta] \circ[\varphi, \varepsilon]$, as in Proposition 3.3(iii), where $\left[{ }_{(C, \varepsilon)}(Y, \eta)_{(B, \beta)}\right]$ is an isomorphism in $\mathcal{A}(G)$ and $\varphi: A \rightarrow M(C)$ is an $\alpha-\varepsilon$ equivariant nondegenerate homomorphism. We shall prove the commutativity of (4.1) by showing that we have two commutative diagrams

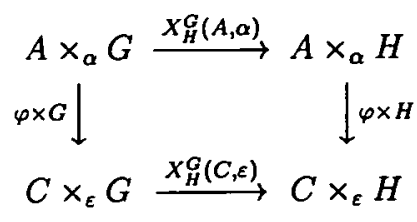


and

$$
\begin{array}{cc}
C \times_{\varepsilon} G \stackrel{X_{H}^{G}(C, \varepsilon)}{\longrightarrow} C \times_{\varepsilon} H \\
\left.Y \times_{\eta} G\right\rfloor \\
B \times_{\beta} G \stackrel{X_{H}^{G(B, \beta)}}{\longrightarrow} B \times_{\beta} H .
\end{array}
$$

Because taking crossed products is a functor, this will then give

$$
\begin{aligned}
{\left[X_{H}^{G}(B)\right] \circ\left[_{A \times G}\left(X \times_{\gamma} G\right)_{B \times G}\right] } & =\left[X_{H}^{G}(B)\right] \circ\left[_{C \times G}\left(Y \times_{\eta} G\right)_{B \times G}\right] \circ[\varphi \times G] \\
& \left.={ }_{C \times H}\left(Y \times_{\eta} H\right)_{B \times H}\right] \circ[\varphi \times H] \circ\left[X_{H}^{G}(A)\right] \\
& \left.={ }_{A \times H}\left(X \times_{\gamma} H\right)_{B \times H}\right] \circ\left[X_{H}^{G}(A)\right],
\end{aligned}
$$

as required.

The commutativity of (4.3) amounts to:

LEMMA 4.2. Suppose $(A, \alpha),(C, \varepsilon)$ are objects in $\mathcal{A}(G)$ and $\varphi: A \rightarrow M(C)$ is a nondegenerate homomorphism such that $\varphi \circ \alpha=\varepsilon \circ \varphi$. Then

$$
X_{H}^{G}(A, \alpha) \otimes_{A \times H}\left(C \times_{\varepsilon} H\right) \cong X_{H}^{G}(C, \varepsilon) \cong\left(C \times_{\varepsilon} G\right) \otimes_{C \times G} X_{H}^{G}(C, \varepsilon)
$$

as right-Hilbert $\left(A \times_{\alpha} G\right)-\left(C \times_{\varepsilon} H\right)$ bimodules.

PROOF: The second isomorphism is standard. For the first, define

$$
\Psi: C_{c}(G, A) \odot C_{c}(H, C) \rightarrow C_{c}(G, C)
$$

by

$$
\Psi(x \otimes g)(t)=\int_{H} \varphi(x(t h)) \varepsilon_{t h}\left(g\left(h^{-1}\right)\right) \Delta(h)^{-1 / 2} d h
$$

The usual change-of-variables arguments show that $\Psi$ is right $C_{c}(H, C)$-linear (though strictly speaking it is not necessary to prove this). The map $\varphi \times H$ is given by

$$
(\varphi \times H(f) g)(h)=\int_{H} \varphi(f(k)) \varepsilon_{k}\left(g\left(k^{-1} h\right)\right) d k
$$

using this identity, some convoluted calculations involving several changes of variables show that

$$
\langle\Psi(x \otimes g), \Psi(y \otimes f)\rangle_{C_{c}(H, C)}=\left(\varphi \times H\left(\langle y, x\rangle_{C_{c}(H, A)}\right) g\right)^{*} f
$$

for $x, y \in C_{c}(G, A) \subset X_{H}^{G}(A)$ and $g, f \in C_{c}(H, C)$. Thus $\Psi$ converts the inner product on the internal tensor product $X_{H}^{G}(A) \otimes_{A \times H}(C \times H)$ to the usual one on $C \times_{\varepsilon} H$.

To see that $\Psi$ has dense range, and hence extends to an isomorphism of right-Hilbert bimodules, it is enough to approximate elements in $C_{c}(G, C)$ of the form $y \cdot f$, where 
$y \in C_{c}(G, C)$ and $f \in C_{c}(H, C)$, with elements in the range of $\Psi$. Moreover, a partition of unity argument shows that functions of the form $t \mapsto \varphi(x(t)) \varepsilon_{t}(c)$ for $x \in C_{c}(G, A)$ and $c \in C$ span an inductive-limit dense - and hence norm-dense - subspace of $C_{c}(G, C)$, so we can assume that $y$ has this form. But now a routine calculation shows that if $g(h)=c f(h)$, then $\Psi(x \otimes g)=y \cdot f$ in $C_{c}(G, C)$; this implies that the range of $\Psi$ is dense.

Another calculation using the analogue of (4.5) for the homomorphism $\varphi \times G$ shows that this isomorphism respects the left action of $A \times_{\alpha} G$.

To prove the commutativity of (4.4), we use a device from [6, Section 4]. Recall that if ${ }_{A} X_{B}$ is an imprimitivity bimodule, then the linking algebra is the collection of $2 \times 2$ matrices

$$
L(X):=\left\{\left(\begin{array}{ll}
a & x \\
\tilde{z} & b
\end{array}\right): a \in A, b \in B, x, z \in X\right\},
$$

with multiplication and involution given by

$$
\left(\begin{array}{ll}
a & x \\
\tilde{z} & b
\end{array}\right)\left(\begin{array}{cc}
a^{\prime} & x^{\prime} \\
\widetilde{z^{\prime}} & b^{\prime}
\end{array}\right):=\left(\begin{array}{cc}
a a^{\prime}+{ }_{A}\left\langle x, z^{\prime}\right\rangle & a \cdot x^{\prime}+x \cdot b^{\prime} \\
\left(z \cdot a^{\prime}+b \cdot z^{\prime}\right)^{\sim} & \left\langle z, x^{\prime}\right\rangle_{B}+b b^{\prime}
\end{array}\right)
$$

and

$$
\left(\begin{array}{cc}
a & x \\
\tilde{z} & b
\end{array}\right)^{*}:=\left(\begin{array}{cc}
a^{*} & z \\
\widetilde{x} & b^{*}
\end{array}\right) .
$$

This has a unique complete $C^{*}$-norm, obtained, for example, by identifying $L(X)$ with the $C^{*}$-algebra $\mathcal{K}(X \oplus B)$ of compact operators on the Hilbert module direct sum $(X \oplus B)_{B}$ (see $[16$, p.50]). The matrices

$$
p=p_{L(X)}:=\left(\begin{array}{cc}
1_{M(A)} & 0 \\
0 & 0
\end{array}\right) \text { and } q=q_{L(X)}:=\left(\begin{array}{cc}
0 & 0 \\
0 & 1_{M(B)}
\end{array}\right)
$$

define full projections in $M(L(X))$ which allow us to identify $A, B$ and $X$ with corners in $L(X)$; we can then use the projections $p, q$ to break up a Hilbert $L(X)$-module into modules over $A$ and $B$. Our key technical result describes some relations among these submodules. It is a mild generalisation of [6, Lemma 4.6].

Propos It ION 4.3. Suppose ${ }_{A} X_{B}$ and ${ }_{C} Y_{D}$ are imprimitivity bimodules, and $Z$ is a right-Hilbert $L(X)-L(Y)$ bimodule. Then

(i) $p Z p=p_{L(X)} Z p_{L(Y)}$ is a right-Hilbert $A-C$ bimodule;

(ii) $q Z q=q_{L(X)} Z q_{L(Y)}$ is a right-Hilbert $B-D$ bimodule;

(iii) $p Z q=p_{L(X)} Z q_{L(Y)}$ is a right-Hilbert $A-D$ bimodule;

(iv) there is an isomorphism $\Phi: X \otimes_{B} q Z q \rightarrow p Z q$ of right-Hilbert $A-D$ modules such that $\Phi(x \otimes z)=x \cdot z$; 
(v) there is an isomorphism $\Psi: p Z p \otimes_{C} Y \rightarrow p Z q$ of right-Hilbert $A-D$ modules such that $\Phi(w \otimes y)=w \cdot y$.

REMARK 4.4. Together, the last two parts of Proposition 4.3 say that the diagram

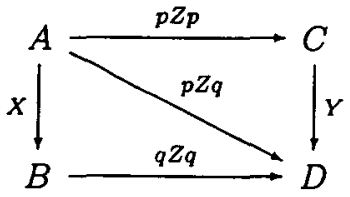

commutes in $\mathcal{C}$. If we had such a proposition when $X$ and $Y$ are just right-Hilbert bimodules, and the linking algebras are by definition $L\left(\mathcal{K}(X)_{X} X_{B}\right)$ and $L\left(\mathcal{K}_{(Y)} Y_{D}\right)$, we could avoid having to factor our morphisms. However, we need to know that $X$ and $Y$ are imprimitivity bimodules to identify the corners $p L(X) p$ and $p L(Y) p$ with $A$ and $C$. Since $C$ in particular occurs in the middle of the internal tensor products in (v), it is hard to see how this hypothesis might be avoided.

LEMmA 4.5. Suppose ${ }_{E} W_{F}$ is a right-Hilbert bimodule and $P \in M(E), Q \in$ $M(F)$ are full projections. Then $P W Q$ is a right-Hilbert $P E P-Q F Q$ bimodule.

Proof: Since $P W Q$ is certainly a $P E P-Q F Q$ submodule, and since

$$
\langle P W Q, P W Q\rangle_{F}=Q\langle P W, P W\rangle_{F} Q \subset Q F Q
$$

we only have to check nondegeneracy of the left action and fullness on the right. For nondegeneracy, we use the fullness of $P$ to see that $P E P \cdot W Q=P E P \cdot E W Q=$ $P(E P E) \cdot W Q$ is dense in $P W Q$. For fullness, we use the fullness of $P$ again to see that

$$
\begin{aligned}
\langle P W Q, P W Q\rangle_{F} & =Q\langle P E \cdot W, P E \cdot W\rangle_{F} Q \\
& =Q\langle W, E P \cdot P E \cdot W\rangle_{F} Q=Q\langle W, E \cdot W\rangle_{F} Q
\end{aligned}
$$

is dense in $Q F Q$.

Proof of Proposition 4.3: For (i), apply Lemma 4.5 with $P=p_{L(X)} \epsilon$ $M(L(X))$ and $Q=p_{L(Y)} \in M(L(Y))$, and note that $A=P L(X) P$ and $C=Q L(Y) Q$ because $X$ and $Y$ are imprimitivity bimodules. Parts (ii) and (iii) follow similiarly.

For part (iv), we first note that because $(x, z) \mapsto x \cdot z$ is bilinear, there is a welldefined map $\Phi$ on the algebraic tensor product $X \odot q Z q$ with the required property. We next verify that $\Phi$ preserves the inner product: if $x, x^{\prime} \in X$ and $z, z^{\prime} \in q Z q$, then the inner product $\left\langle x, x^{\prime}\right\rangle_{B}$ is given in $L(X)$ by $\left\langle x, x^{\prime}\right\rangle_{B}=x^{*} x^{\prime}$; more formally,

$$
\left(\begin{array}{cc}
0 & 0 \\
0 & \left\langle x, x^{\prime}\right\rangle_{B}
\end{array}\right)=\left(\begin{array}{ll}
0 & x \\
0 & 0
\end{array}\right)^{*}\left(\begin{array}{cc}
0 & x^{\prime} \\
0 & 0
\end{array}\right) .
$$


Thus

$$
\begin{aligned}
\left\langle x \otimes z, x^{\prime} \otimes z^{\prime}\right\rangle_{D} & =\left\langle z,\left\langle x, x^{\prime}\right\rangle_{B} \cdot z^{\prime}\right\rangle_{D} \\
& =\left\langle z,\left(x^{*} x^{\prime}\right) \cdot z^{\prime}\right\rangle_{D} \\
& =\left\langle x \cdot z, x^{\prime} \cdot z^{\prime}\right\rangle_{D} \\
& =\left\langle\Phi(x \otimes z), \Phi\left(x^{\prime} \otimes z^{\prime}\right)\right\rangle_{D},
\end{aligned}
$$

and $\Phi$ extends to an isometry of $X \otimes_{B} q Z q$ into $p Z q$. To see that $\Phi$ has dense range and is therefore onto, note that $L(X)$ acts nondegenerately on $Z$, so

$$
\text { range } \Phi \supset p L(X) q \cdot q Z q=p L(X) q \cdot q(L(X) \cdot Z) q=p(L(X) q L(X)) \cdot Z q
$$

is dense because $q$ is full.

For (v), note that the $D=q L(Y) q$-valued inner product is given by the product in $L(Y)$, so

$$
\begin{aligned}
\left\langle w \otimes y, w^{\prime} \otimes y^{\prime}\right\rangle_{D} & =\left\langle y,\left\langle w, w^{\prime}\right\rangle_{C} \cdot y^{\prime}\right\rangle_{D} \\
& =y^{*}\left\langle w, w^{\prime}\right\rangle_{L(Y)} y^{\prime} \\
& =\left\langle w \cdot y, w^{\prime} \cdot y^{\prime}\right\rangle_{L(Y)}
\end{aligned}
$$

which is $\left\langle w \cdot y, w^{\prime} \cdot y^{\prime}\right\rangle_{D}$ because $w \cdot y$ and $w^{\prime} \otimes y^{\prime}$ belong to $p Z q$. Thus $w \otimes y \mapsto w \cdot y$ extends to an isometry $\Psi$, as claimed, and $\Psi$ is surjective because $p$ is full and $L(Y)$ acts nondegenerately on $Z$.

BACK TO THE PROOF OF THEOREM 4.1. Consider the action $\delta=\left(\begin{array}{ll}\varepsilon & \eta \\ \tilde{\eta} & \beta\end{array}\right)$ of $G$ on $L(Y)$; by Green's theorem, $Z=X_{H}^{G}(L(Y))$ is then a right-Hilbert $\left(L(Y) \times_{\delta} G\right)$ $\left(L(Y) \times_{\delta} H\right)$ bimodule. To show that (4.4) commutes, we aim to apply Proposition 4.3 to $Z$, but this requires that we first identify $L(Y) \times_{\delta} G$ with $L\left(Y \times_{\eta} G\right)$, and similarly for $H$. Now the dense subalgebra $C_{c}(G, L(Y))$ consists of $2 \times 2$ matrices with entries in $C_{c}(G, C), C_{c}(G, Y)$, et cetera, and the diagonal corners have their usual *-algebraic structures as subalgebras of $C \times_{\varepsilon} G$ and $B \times{ }_{\beta} G$. The norms are the same too: every covariant representation of $(L(Y), \delta)$ restricts to a covariant representation of $(B, \beta)$, and the inducing construction of Example 2.8 shows that every covariant representation of $(B, \beta)$ extends to a covariant representation of $(L(Y), \delta)$. So we have embeddings of $C \times{ }_{\varepsilon} G$ and $B \times{ }_{\beta} G$ as corners in $L(Y) \times{ }_{\delta} G$. The bimodule crossed product $Y \times{ }_{\eta} G$ also embeds: because $B \times G$ embeds isometrically, the norm of a function

$$
t \mapsto\left(\begin{array}{cc}
0 & f(t) \\
0 & 0
\end{array}\right) \in C_{c}(G, L(Y)) \subset L(Y) \times G
$$

is just $\left\|\langle f, f\rangle_{B \times G}\right\|$. These embeddings combine to give an isomorphism of $L\left(Y \times_{\eta} G\right)$ onto $L(Y) \times_{\delta} G$, as desired. (This observation is not new: in [2], Combes defines $Y \times_{\eta} G$ to be the corner in $L(Y) \times_{\delta} G$.) In exactly the same way, $L\left(Y \times_{\eta} H\right) \cong L(Y) \times_{\delta} H$. 
We next have to identify the corners $p X_{H}^{G}(L(Y), \delta) p$ and $q X_{H}^{G}(L(Y), \delta) q$ with $X_{H}^{G}(C, \varepsilon)$ and $X_{H}^{G}(B, \beta)$. But viewing elements of $C_{c}(G, L(Y)) \subset X_{H}^{G}(L(Y), \delta)$ as matrices of functions, and similarly for $C_{c}(G, L(Y)) \subset L(Y) \times_{\delta} G$ and $C_{c}(H, L(Y)) \subset$ $L(Y) \times_{\delta} H$, gives the desired identifications. It now follows from Proposition 4.3 that

$$
\left(Y \times_{\eta} G\right) \otimes_{B \times G} X_{H}^{G}(B, \beta) \cong p X_{H}^{G}(L(Y), \delta) q \cong X_{H}^{G}(C, \varepsilon) \otimes_{C \times H}\left(Y \times_{\eta} H\right)
$$

as right-Hilbert $\left(C \times{ }_{\varepsilon} G\right)-\left(B \times{ }_{\beta} H\right)$ bimodules. In other words, (4.4) commutes in the category $\mathcal{C}$.

We have now established the first part of Theorem 4.1, the natural transformation between the functors $(A, \alpha) \mapsto A \times_{\alpha} G$ and $(A, \alpha) \mapsto A \times_{\alpha} H$. For the second part, we have to prove that (4.2) commutes. We follow the same procedure as before: factor a given morphism $\left[{ }_{A} X_{B}, \gamma\right]$ as $\left[{ }_{C} Y_{B}, \eta\right] \circ[\varphi, \varepsilon]$, and prove that two separate diagrams commute. To see the commutativity of

$$
\begin{array}{lr}
\left(A \otimes C_{0}(G / H)\right) \times_{\alpha \otimes \tau} G \stackrel{X_{H}^{G(A, \alpha)}}{\longrightarrow} A \times_{\alpha} H \\
(\varphi \otimes \mathrm{did}) \times G \mid & \left.\right|_{\varphi \times H} \\
\left(C \otimes C_{0}(G / G)\right) \times_{\varepsilon \otimes \tau} G \stackrel{X_{H}^{G}(C, \varepsilon)}{\longrightarrow} C \times_{\varepsilon} H,
\end{array}
$$

we merely note that the isomorphism of Lemma 4.2 respects the left action of $C_{0}(G / H)$, and hence is also an isomorphism of right-Hilbert $\left(\left(A \otimes C_{0}(G / H)\right) \times_{\alpha \otimes \tau} G\right)-\left(C \times_{\varepsilon} H\right)$ bimodules. To see that

$$
\begin{array}{cc}
\left(C \otimes C_{0}(G / H)\right) \times_{\varepsilon \otimes \tau} G & \stackrel{X_{H}^{G}(C, \varepsilon)}{\longrightarrow} C \times_{\varepsilon} H \\
\left.\left(Y \otimes C_{0}(G / H)\right) \times_{\eta \otimes r} G\right\rfloor & \downarrow Y \times_{\eta} H \\
\left(B \otimes C_{0}(G / H)\right) \times_{\beta \otimes \tau} G \stackrel{X_{H}^{G}(B, \beta)}{\longrightarrow} B \times_{\beta} H
\end{array}
$$

commutes, we again apply Proposition 4.3 to $Z=X_{H}^{G}(L(Y), \delta)$, this time viewed as a $L\left(\left(Y \otimes C_{0}(G / H)\right) \times_{\eta \otimes \tau} G\right)-L\left(Y \times_{\eta} H\right)$ imprimitivity bimodule. To do so, we have to identify $L\left(\left(Y \otimes C_{0}(G / H)\right) \times_{\eta \otimes \tau} G\right)$ with $\left(L(Y) \otimes C_{0}(G / H)\right) \times_{\delta \otimes \tau} G$, but there is no essential difference in the argument.

This concludes the proof of our main theorem.

REMARK 4.6. As an example of how the present set of ideas can simplify things, notice that the commutativity of diagram (4.4) implies that for each covariant representation $\rho \times V$ of $B \times{ }_{\beta} H$, the representation $\left(Y \times_{\eta} G\right)$-Ind( $\left.\operatorname{Ind}_{H}^{G}(\rho \times V)\right)$ is equivalent to $\operatorname{Ind}_{H}^{G}\left(\left(Y \times_{\eta} H\right)-\operatorname{Ind}(\rho \times V)\right)$. This is the content of [4, Theorem 3], or at least the content of its proof (the twists in that theorem go along for free by [4, Remark (2) on p.174] and [7, Corollary 5]). The point is that the arguments we give here are more elegant as well as more powerful. 


\section{REFERENCES}

[1] R. Curto, P. Muhly and D. Williams, 'Cross products of strongly Morita equivalent $C^{*}$-algebras', Proc. Amer. Math. Soc. 90 (1984), 528-530.

[2] F. Combes, 'Crossed products and Morita equivalence', Proc. London Math. Soc. 49 (1984), 289-306.

[3] A. Connes, Noncommutative geometry (Academic Press, San Diego, 1994).

[4] S. Echterhoff, 'Morita equivalent twisted actions and a new version of the Packer-Raeburn stabilization trick', J. London Math. Soc. 50 (1994), 170-186.

[5] S. Echterhoff and I. Raeburn, 'Multipliers of imprimitivity bimodules and Morita equivalence of crossed products', Math. Scand. 76 (1995), 289-309.

[6] S. Echterhoff and I. Raeburn, 'The stabilisation trick for coactions', J. reine angew. Math. 470 (1996), 181-215.

[7] P. Green, 'The local structure of twisted covariance algebras', Acta Math. 140 (1978), 191-250.

[8] G.G. Kasparov, 'Equivariant $K K$-theory and the Novikov conjecture', Invent. Math. 91 (1988), 147-201.

[9] G.G. Kasparov, ' $K$-theory, group $C^{*}$-algebras, and higher signatures (conspectus)', in Novikov conjectures, index theorems, and rigidity. Vol. 1 (Oberwolfach, 1993), London Math. Soc. Lecture Note Ser. 226 (Cambridge University Press, Cambridge, 1995), pp. 101-146.

[10] S. Kaliszewski, J. Quigg and I. Raeburn, 'Duality of restriction and induction for $C^{*}$-coactions', Trans. Amer. Math. Soc. 349 (1997), 2085-2113.

[11] E.C. Lance, Hilbert $C^{*}$-modules, London Math. Soc. Lecture Note Ser. 210 (Cambridge University Press, Cambridge, 1995).

[12] E. Mendelson, Introduction to mathematical logic (Van Nostrand, Princeton, NJ, 1964).

[13] P. Muhly and B. Solel, 'Tensor algebras over $C^{*}$-correspondences: representations, dilations, and $C^{*}$-envelopes', J. Funct. Anal. 158 (1998), 389-457.

[14] M.V. Pimsner, 'A class of $C^{*}$-algebras generalizing both Cuntz-Krieger algebras and crossed products by Z.', in Free Probability Theory 12, (D.-V. Voiculescu, Editor), Fields Inst. Commun. (American Mathematical Society, Providence, RI, 1997), pp. 189-212.

[15] I. Raeburn, 'On crossed products and Takai duality', Proc. Edinburgh Math. Soc. 31 (1988), 321-330.

[16] I. Raeburn and D.P. Williams, Morita equivalence and continuous-trace $C^{*}$-algebras (American Mathematical Society, Providence, RI, 1998).

[17] M. Takesaki, 'Covariant representations of $C^{*}$-algebras and their locally compact automorphism groups', Acta Math. 119 (1967), 273-303. 
Fachbereich Mathematik und Informatik University of Münster 48149 Münster

Germany

e-mail: echters@uni-muenster.de

Department of Mathematics

Arizona State University

Tempe AZ 85287

United States of America

e-mail: quigg@math.la.asu.edu
Department of Mathematics

Arizona State University

Tempe AZ 85287

United States of America

e-mail: kaz@math.la.asu.edu

Department of Mathematics

University of Newcastle

New South Wales 2308

Australia

e-mail: iain@maths.newcastle.edu.au 